\title{
Cross-Layer Optimization of AODV Routing Protocol For Mobile Ad-Hoc Network (MANET)
}

\author{
Muhammed Kamrul Islam \\ School of Electronic Information and Engineering \\ Beihang University, PR China \\ e-mail: kamrul8766@yahoo.com
}

\author{
Rong Ke Liu \\ School of Electronic Information and Engineering \\ Beihang University, PR China \\ e-mail: rongke_liu@buaa.edu.cn
}

\begin{abstract}
Mobile Ad hoc Networks (MANETs) are temporary formed, infrastructure-less networks. The performance metrics deteriorate due to the unstable channel conditions, network connectivity, mobility and resource limitations. To improve different performance metrics, various cross-layering approaches are utilized where different OSI layer information are exchanged. AODV is a popular distance vector proactive routing algorithm. In our research we investigate a modified version of AODV routing protocol, based on route discovery by utilizing Physical Layer information instead of the minimum hop count approach of the default distance vector algorithm. The research also elaborates how the proposed model uses the received SNR to find its route. The paper also proposes future research direction for utilizing Polar Coding for fading channel. The results of the simulations show improvement on the existing default AODV performance metrics like MANET traffic throughput, application specific response time, data dropped, delay etc.
\end{abstract}

Keywords- AODV, Cross-Layer, MANET, Routing

\section{INTRODUCTION}

Mobile ad hoc network (MANET) consisting of mobile hosts only has attracted much attention recently. In MANET, the nodes themselves are responsible for routing and forwarding of packets. If the nodes are out of range from each other, and therefore are not able to communicate directly, intermediate nodes are needed to make up the network in which the packets are to be transmitted. Examples of MANETs include emergency operations where there exis no infrastructure and military operations where the existing infrastructure might not be trusted.

The design of efficient routing protocols is a critical issue for MANET having no fixed topology. Therefore, the source-initiated on demand routing protocol, which establishes the route between the source and the destination only when the source demands that, becomes the most popular routing protocol in the MANET.

The layered concept (for example OSI) was primarily created for wired networks and naturally follows their architectural design. Designing wireless networks with strict layering principle did not fulfill the expectation raised in wire-line network design. The ad hoc mobile networks oppose strict layered protocol design because of their dynamic nature, infrastructure-less architecture, limited resources, mobility of nodes and time varying unstable links and topology. The concept of cross-layer design is based on architecture where the layers can exchange information in order to improve the overall network performances [1].

\section{MANET \& Routing Protocol}

The term MANET stands for Mobile Ad-hoc Network. Ad hoc wireless networks are defined as the category of wireless networks that utilize multi-hop radio relaying and are capable of operating without the support of any fixed infrastructure (infrastructure less networks). The absence of any central coordinator or base station makes the routing a complex one compared to cellular networks. Each node acts as a host and a router at the same time. This means that each node participating in a MANET commits itself to forward data packets from a neighboring node to another until a final destination is reached. These networks are deployed 'on the fly' [2][3].

Among the issues of Adhoc Wireless Networks, routing is one of the key features. Since the network is dynamic and channel state is continuously changing, discovering the appropriate path for data transfer is very important. The MAC for $802.11 \mathrm{~b}$ uses CSMA/CA for accessing the channels by different nodes. But, one of the major concerns is not only selecting the nodes along with the path from source to destination but also pick those nodes in such a way that, they provide best service in the form of relaying data with high rate, least error and least time. As such, this research mainly focuses on the routing issue.

\section{Classification of Routing Protocols}

Routing protocols for MANETs can be classified into several types based on different criteria. Based on routing information update mechanism, they can be categorized as follows [4]:

\section{A. Table Driven-Proactive}

Every node maintains the network topology information in the form of routing tables by periodically exchanging routing information. The routing information is generally flooded in the network. Whenever a node requires a path to a destination, it runs in an appropriate path finding algorithm on the topology information it maintains. Examples of such protocols are DSDV, OLSR, WRP etc. Such protocols have both advantages and disadvantages eg. Availability of routes to all destinations at all times facilitates route setup quickly. But, it has excessive control overhead which is proportional to the number of nodes in network. 


\section{B. Reactive or On-demand routing protocols}

Such protocols do not maintain the network topology information. They only obtain the necessary path on requirement basis. As a result these protocols do not exchange routing information periodically. Examples are: DSR, AODV etc. While such protocols may be taking time to establish routing path, they have much lesser control overhead. They are the classical distance vector protocols.

\section{Selection of Protocol: AODV}

The table driven proactive routing protocols have the advantage of having an available route always ready to the destination. But it comes with cost of consuming a big part of the bandwidth resource, most of which may not even be used. Thus, an appropriate routing protocol for MANETs should imply a reasonable over-head in order to preserve the limited bandwidth. Message complexity must be kept very low. On the other hand, the reactive routing protocol reduces the overhead traffic by creating a route only when it is required. When a route is no longer used in reactive protocols, it is simply expunged from the routing table. For these reasons reactive protocols are of more interest for the MANET community as reactive protocols[4].

Considering the aforesaid facts, our research selected Ad Hoc On-Demand Distance Vector (AODV) protocol for improving performance.

Ad Hoc On-Demand Distance Vector (AODV) Routing Protocol uses an on-demand approach for finding routes. As a result, a route is established only when it is required by a source node for transmitting data packets. It employs destination sequence numbers to identify the most recent path. The source node and the intermediate nodes store the next hop information corresponding to each flow for data packet transmission [3]. In AODV, each routing table entry contains the following information:

- Destination

- Next hop

- Number of hops

- Destination sequence number

- Active neighbours for this route

- Expiration time for this route table entry

Expiration time, also called lifetime, is reset each time the route has been used. The new expiration time is the sum of the current time and a parameter called active route timeout. RFC 3561 defines it to 3 seconds.

\section{SNR BASED AODV}

Keeping the trade-off concern in mind, our research applied the 'lower to upper layers' approach where information from physical layer is utilized in the Network Layer where least complexity for calculation would be required with the available channel side information. The physical layer is responsible for transmission of bits aiming to achieve minimum bit error rate. The most relevant metrics that describe the physical layer features are bit error rate (BER) and SNR (signal-to-noise ratio). Also, SNIR (signal- to-noise-interference ratio) captures the interference effect from the environment. Another important parameter is the transmitting power (depends on the operation mode) and battery status. Particular physical layer design and information gained from physical layer conditions can influence the upper layer solutions. On the other hand, the user/application requirements can require particular physical operation mode [1].

The proposed model assumes that, during the route discovery process, each node has the channel side information available in terms of received SNR in that packet transmission. While a node receives the route request, it also has the information of the SNR. If the node takes part in the route reply process, then it stores the SNR value in the buffer. During the reverse path setup of routes, the SNR values along. A similar approach was also proposed in [5] for DSR protocol, but we additionally proposed the methodology of adopting the SNR based approach for AODV protocol with respect to suitability of application and also some future research reports on Polar coding to be implemented in the cross-layer design.

\section{PRoposed ModeL}

It is assumed that, in the MANET, once a node receives a packet (eg. Route request), it has the channel side information available in terms of received SNR. For example, if a route request is received from i to $j, \operatorname{SNR}(i, j)$ is available at node $\mathrm{j}$.

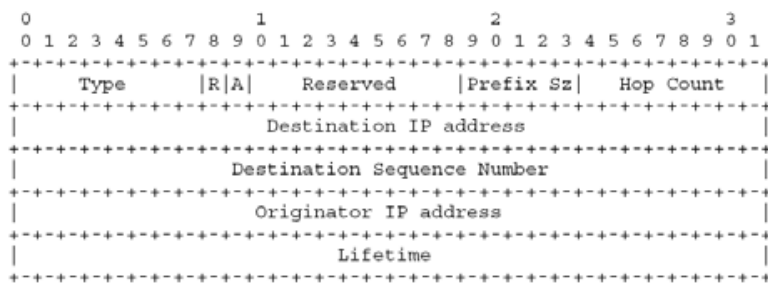

Figure 1 : AODV Route Reply Packet Format

It may be mentioned that, there are 9 reserved bits in the packet, which is set to 0 in AODV operation.

In order to embed the SNR information in the route reply process we propose the usage of reserve bits as shown in Figure 2.

\begin{tabular}{|l|l|l|l|l|l|l|l|l|}
\hline 0 & 1 & 2 & 3 & 4 & 5 & 6 & 7 & 8 \\
\hline $\mathrm{T}$ & \multicolumn{1}{|l|}{ SNR value } \\
\hline
\end{tabular}

Figure 2: AODV Route Reply packet Reserve bits allocation

$\mathrm{T}=1$ bit: Toggle bit 1 or 0 ; If 1 , then the proposed SNR based AODV will be activated and if 0 then traditional default AODV will be activated.

$\mathrm{SNR}=8$ bits: puts the received $\mathrm{SNR}$ at node $\mathrm{j}$ from a transmission of route request from $(\mathrm{i}, \mathrm{j})$ as described below.

Steps of route discovery for SNR based AODV 
The following steps show the route discovery process of the proposed SNR based AODV:

- A route is required to be established from source to destination. The nodes in the network will know to put $\mathrm{T}=1$ to activate SNR based AODV protocol in the route reply packet.

- The neighbor nodes of source (and so on) receive route request packet. It also has the information on the received SNR of the packet during reception.

- The node does the following in respective options:

(i) If the node itself is the destination, it puts the received SNR value in the route reply packet (reserved bits as mentioned earlier).

(ii) If the node is to rebroadcast the route request to its neighbours, it stores the SNR value in its buffer for future use.

(iii) If the node is neither the destination, nor is to rebroadcast the route request, it discards the received SNR value.

- With the relay method, the route request reaches the destination. (i) above is executed.

- In the reverse path transmission of route reply, each pair of nodes compares the SNR values 'stored in the buffer' and 'received via route reply'. Only the smaller value of the two is stored in the next route reply reverse path. The process continues till reaching back to source node.

- The source node receives 1 or multiple routing paths. For multiple routing paths, each route reply packet for each path has the lowest available SNR value available through that path. Instead of minimum hop count, the source chooses the path with higher value of SNR (which is among the lower of each path).

- The data starts transmitting in the newly defined higher SNR path.

\section{Simulation Results}

OPNET Modeler was used for the simulation purpose of the research. Different controlled scenarios were simulated to observe the performance of the SNR based AODV. Some of the simulation scenarios are presented in this section.

TABLE I. MANET SIMULATION PARAMETERS

\begin{tabular}{|l|l|}
\hline \multicolumn{1}{|c|}{ Parameters } & \multicolumn{1}{c|}{ Value } \\
\hline Area & $1 \mathrm{~km} \mathrm{X} 1 \mathrm{~km}$ \\
\hline Nodes & $25(10 \mathrm{~m} / \mathrm{s})$ \\
\hline Traffic & Raw MANET Traffic (S-D) \\
\hline Protocol & AODV default \& custom (2 scenarios) \\
\hline MAC & $802.11 \mathrm{~b}$ CSMA/CA \\
\hline Node Range & $300 \mathrm{~m}$ \\
\hline
\end{tabular}

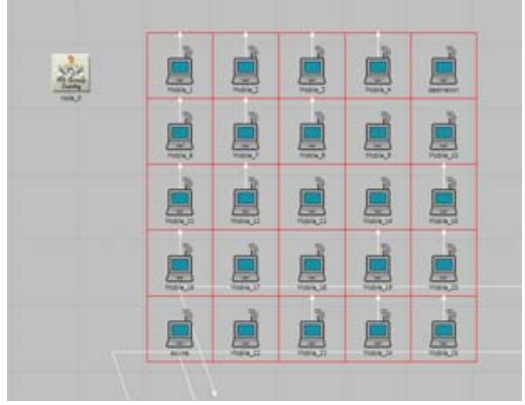

Figure 3 : MANET Traffic Simulation Scenario

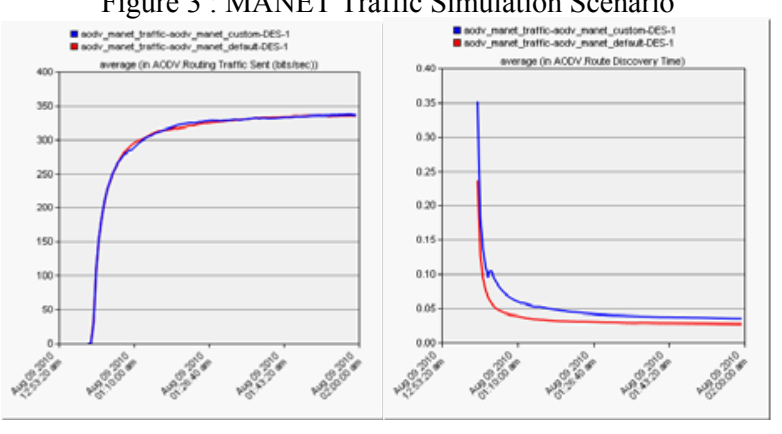

Figure.4a: Routing Traffic Sent Figure 4 b: Routing Discovery Time

Figure $4 \mathrm{a}$ shows that the routing traffic sent is similar for both the protocols on average. But, Figure $4 \mathrm{~b}$ shows that default AODV has less route discovery time which is also expected since the SNR-based AODV takes more hops. However, the following graphs show the trade-off which is achieved by the SNR-based AODV at the cost of little higher routing discovery time.

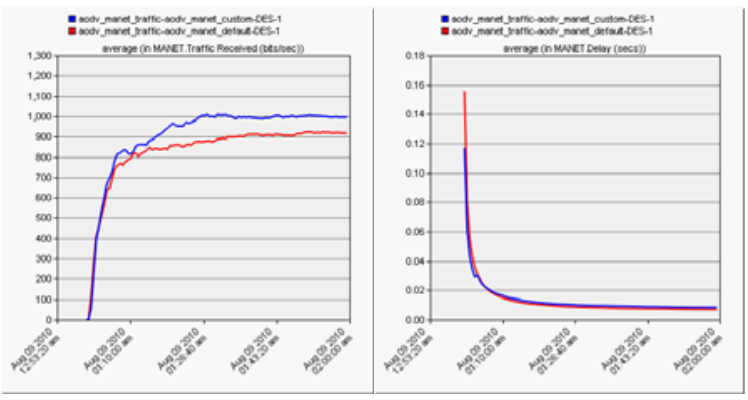

Fig.5a: MANET Traffic Rx by Dest Fig.5b: Average MANET Delay

Figure 5a shows that the SNR-based AODV received more MANET traffic at the destination which leads to more throughput. Figure 5b shows that even to achieve this more throughput, the MANET delay was same in the default AODV and the custom SNR-based AODV protocol. 
TABLE II. FTP SIMULATION PARAMETERS

\begin{tabular}{|l|l|}
\hline Parameters & Value \\
\hline Area & $2 \mathrm{~km} \mathrm{X} 2 \mathrm{~km}$ \\
\hline Nodes & $20(5 \mathrm{~m} / \mathrm{s})$ \\
\hline Application & FTP \\
\hline Protocol & AODV default \& Custom \\
\hline Traffic rate & $11 \mathrm{Mbps}$ \\
\hline Packet Size & $1024 \mathrm{bits}$ \\
\hline Carrier Freq & $2.4 \mathrm{GHz}$ \\
\hline
\end{tabular}

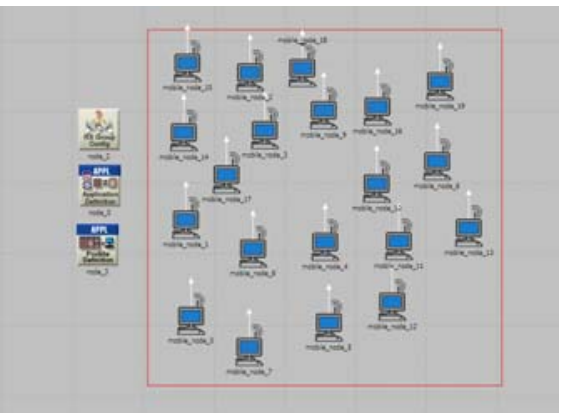

Figure 6: FTP application Simulation Scenario

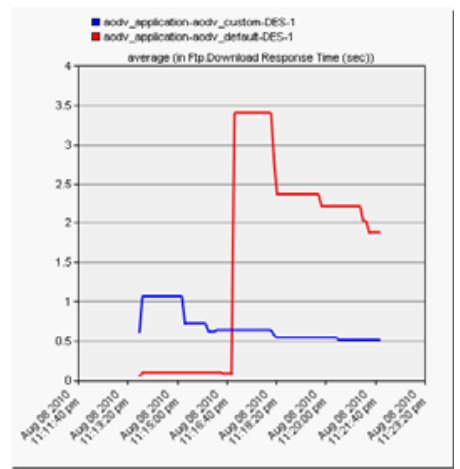

Figure 7: FTP Download Response Time

Figure 7 shows much lower download response time for custom AODV which is a desirable parameter for FTP application.

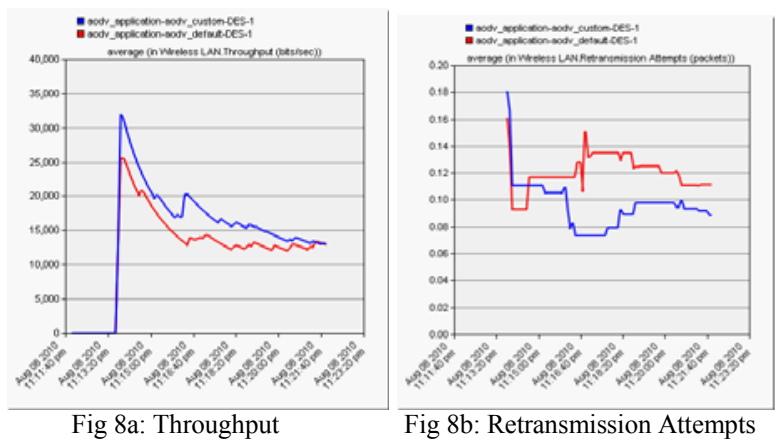

Figure 8a shows that the SNR-based AODV has better throughput than the default AODV protocol. Figure $8 \mathrm{~b}$ shows that, due to link stability in the SNR-based AODV, the retransmission attempts are much less than that of default AODV. This in return helps the network to have less routing overhead and sending the same data again, and thereby consuming the bandwidth.

Hence, it is observed that, the SNR-based AODV protocol outperforms the default AODV routing protocol in some of the major performance metrics as shown. It is also observed that, some of the data applications, the stability of the link helps to achieve higher throughput in the SNR-based AODV protocol.

\section{CONCLUSION \& FUTURE WORKS}

MANETs are infrastructure-less networks where all the nodes act as host as well as routes to deliver data. By the nature and the architecture, the performance is affected by channel conditions, network connectivity, mobility and resource limitations. Various cross-layering approaches are utilized to improve the performance of MANETs and their associated routing protocols. Our research investigated a modified version of AODV routing protocol utilizing Physical Layer information i.e. SNR. The proposed model uses the received SNR to find its route instead of the default hop count mechanism of AODV protocol. The simulation of proposed SNR based AODV shows performance improvement of the proposed SNR-based AODV protocol.

To improve the cross-layer performance further, we report here the work in progress i.e. incorporating the channel coding. Polar coding as proposed in [6] has been proven to be capacity achieving for Binary-Discrete Memory-less Channel (B-DMC). Our research focus on use Polar Coding for a type of wireless channel namely fast Fading Channel. As per the B-DMC channel and Polar coding, the channel needs to be memoryless as well as ergodic. But, the fast fading Rayleigh channel is neither memoryless nor ergodic. But, we still are interested to take the advantage of the capacity achieving feature of Polar Coding that can deliver us good performance (e.g. in terms of ber) for the wireless communication channel. To do that, we follow certain assumptions and approximations to model the channel as a set of parallel independent BI-AWGN channel and use the polar coding scheme.

\section{REFERENCES}

[1] Liljana Gavrilovska, -Cross-Layering Approaches in Wireless Ad Hoc Networks Wireless Personal Communications (2006) 37: 271-290

[2] Kenneth Holter, Comparing AODV and OLSR, report, 23rd April 2005

[3] Lyes Guemari, -An OPNET Model implementation for Ad-hoc On Demand Distance vector Routing Protocol, Institute National des Telecommunications, Paris - France, August 20, 2001.

[4] C. Siva Ram Murthy, BS Manoj, -Ad-hoc Wireless Networks, Architecture and Protocol, Pearson Education, ISBN-978-8-317-0688-6, 2008.

[5] Fuad Alnajjar, Yahao Chen, -SNR/RP AWARE ROUTING ALGORITHM: CROSS-LAYER DESIGN FOR MANETS\|, International Journal of Wireless \& Mobile Networks (IJWMN), Vol 1, No 2, November 2009.

[6] E. Arikan and S. Member, "Channel Polarization: A Method for Constructing Capacity-Achieving Codes for Symmetric Binary-Input Memoryless Channels," IEEE Transactions on Information Theory: vol. 55 , no. 7, pp. 3051-3073, 2009. 